\title{
High Purity Metals as Primary Calibration Materials for Elemental Analysis-Their Importance and Their Certification
}

\author{
Ralf Matschat, Michael Czerwensky, Sandra Pattberg, Hans-Joachim Heinrich and Silke Tutschku
}

BAM, Federal Institute for Materials Research and Testing, D-12200 Berlin, Germany

The Bundesanstalt für Materialforschung und -prüfung, BAM (Federal Institute for Materials Research and Testing) continues to establish a system of primary reference materials to meet the demands of metrological traceability. The materials act as national standards in the field of elemental analysis. For all elements of the periodic table —excepting those that are gases or radioactive - two different kinds of reference materials are being certified. The substances are of very high purity and of defined stoichiometry. Pure elements and metals are used as far as possible. They are certified by determining the trace contents of most elements of the periodic table at very low levels using different trace element analysis methods. Recent application of these methods is described and examples of the certification of some pure metals (copper, iron and lead) are given.

(Received August 3, 2001; Accepted November 26, 2001)

Keywords: Trace elemental analysis, atomic spectrometry, high purity metals, certification, primary calibration materials, reference materials, national standards

\section{Introduction}

"Non-primary" analytical methods needing a calibration, such as atomic absorption spectrometry (AAS), inductively coupled plasma optical emission spectrometry (ICP-OES), or inductively coupled plasma mass spectrometry (ICP MS) are used world-wide in most laboratories for tasks of elemental analysis today. This explains their very high importance. They need a common primary metrological basis for the traceability of their calibration. The calibration of these methods of elemental analysis with liquid sample handling is carried out by using calibration solutions, in which the concentration of analytes are set to different values. Generally the matrix concentration of calibration solutions is adjusted to matrix concentration of solutions to be analysed.

A schematic overview of a current trend in elemental analysis is shown in Fig. 1. This trend leads to an increasing propagation of atomic spectrometric methods needing certified reference materials with special "natural" matrix composition ("matrix CRMs") for their verification of trueness. For the traceability of the method calibration matrix CRMs or primary pure materials (to prepare calibration solutions) are needed.

The relation of matrix CRMs to certified high purity materials is described in Fig. 2. The different cases in which certified high purity materials are needed as basic materials for the traceability in the process of certification of matrix CRMs are specified. For the importance of matrix CRMs themselves see Fig. 1.

To calibrate the mass fraction of an analyte a pure substance with a defined uncertainty of the mass fraction of its main component (the analyte) is needed, which we call Type A material. The uncertainty is directly transferred to the uncertainty of the final analytical result. Therefore the great importance of solid pure substances used for the calibration or for the preparation of calibration solutions is evident. The uncertainty of the mass fraction of the pure substance should be about one order of magnitude lower than the tolerable un-

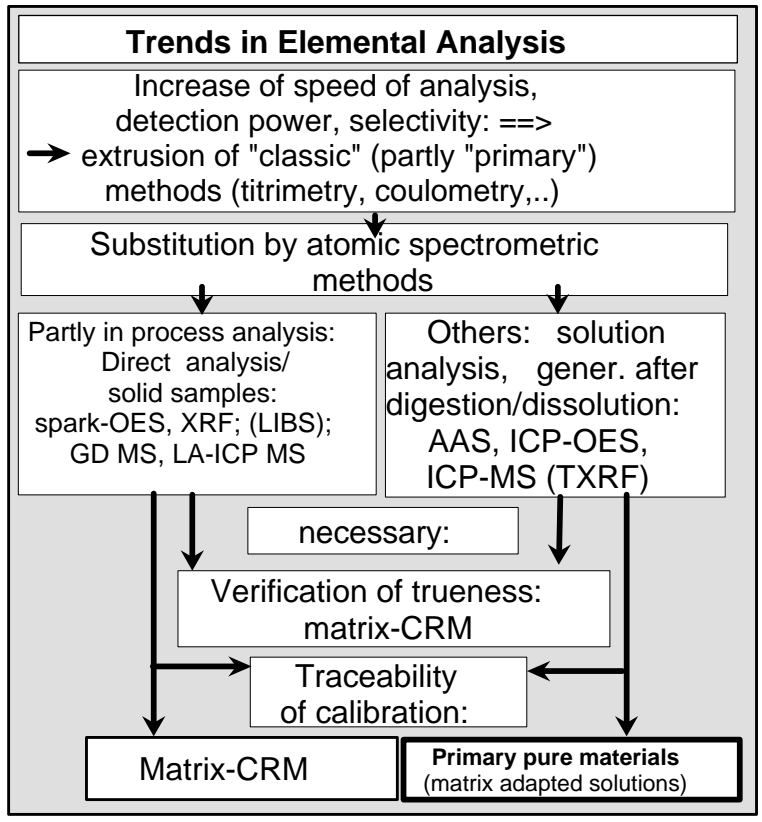

Fig. 1 Scheme of some important trends in modern elemental analysis demonstrating the importance of certified primary pure materials and of certified reference materials for specific matrices (matrix CRMs).

certainty of the final analytical result in order to avoid contribution to its uncertainty significantly. In metals analysis and especially in precision analysis of higher contents rather low combined uncertainties for the final result down to about $0.1 \%$ are often demanded. Thus the uncertainty of the main component of a primary Type A material for analyte calibration should not exceed $0.01 \%$. Certified pure materials with such a low uncertainty of the mass fraction of the main component are not on the market and must be certified.

We have to clarify that the purity mark of pure metals is normally based on the sum of all determined "metallic" impurities which is subtracted from $100 \%$. This does not indicate the real purity because some metallic traces and all the non-metallic traces are not taken into account. Therefore for 


\begin{tabular}{|c|c|c|c|}
\hline \multicolumn{4}{|c|}{ Certification of matrix CRMs } \\
\hline \multicolumn{4}{|c|}{$\begin{array}{l}\text { Traceability to SI unit } \\
\text { in the step of final determination }\end{array}$} \\
\hline \multicolumn{2}{|c|}{$\begin{array}{l}\text { classical "primary" } \\
\text { methods and IDMS } \\
\text { (But: validation, } \\
\text { or traceability } \\
\text { often using certified } \\
\text { high purity materials }\end{array}$} & \multicolumn{2}{|c|}{$\begin{array}{l}\text { liquid sample } \\
\text { methods to be } \\
\text { calibrated with } \\
\text { relation to } \\
\text { certified high } \\
\text { purity materials }\end{array}$} \\
\hline \multicolumn{4}{|c|}{ Checking trueness of digestion } \\
\hline $\begin{array}{c} \\
\text { solid sample } \\
\text { direct methods } \\
\text { such as } \\
\text { INAA,IPAA, } \\
\text { calibrated with } \\
\text { certified } \\
\text { high } \\
\text { purity } \\
\text { materials }\end{array}$ & \begin{tabular}{|c|} 
inclusion \\
of digestion \\
into IDMS \\
measurement \\
Backspike: \\
certified \\
high \\
purity \\
materials
\end{tabular} & $\begin{array}{c}\downarrow \\
\text { recovery } \\
\text { experiments } \\
\text { with } \\
\text { certified } \\
\text { high } \\
\text { purity } \\
\text { materials }\end{array}$ & \begin{tabular}{|c} 
matrix- \\
CRM ? \\
(vali- \\
dated \\
digestion?) \\
$? ?$ \\
not \\
possible
\end{tabular} \\
\hline
\end{tabular}

Fig. 2 Scheme of the certification of matrix CRMs in relation to certified high purity materials.

pure metals, we should distinguish between the terms " $\mathrm{mxN}$ ", (with "m" standing for "metallic") and "txN", (with "t" standing for "total"). We found out that there is a lack of defined pure substances with "t-purity" having a defined and low uncertainty for acting as a starting point for the traceability chain of elemental analysis. Therefore BAM has decided to develop a system of primary calibration substances for analyte calibration (called "Type A") and for matrix adaption (called "Type B"). The uncertainty for the mass fraction of the main component of Type A materials should not exceed $0.01 \%$. This upper limit of uncertainty meets the highest demands from precision analysis and the use of these substances for preparing primary natural backspike solutions for isotope dilution mass spectrometry (IDMS).

According to a contract between the national institutes of Germany, Physikalisch-Technische Bundesanstalt, (PTB) and BAM the objective of the system of primary calibration substances is to represent the "National Standards in the field of Elemental Analysis". From these substances very well certified primary solutions will be prepared by these institutions. For the future, the co-operation with national institutes of other countries is intended. The primary calibration system includes two types of highly pure substances for each element of the periodic system, -excepting those that are gases or radioactive. If possible, pure elements are used to avoid additional problems of stoichiometry. The kind of substances described in this article (Type A) is assigned for the traceability of analyte calibration, and might be used for problems of determining recovery and as back-spikes for the certification of spike materials used for the primary method of IDMS. The other type of material (Type B) is for metrological aspects of matrix adaption and is not discussed in this article. For further information see. ${ }^{1)}$

Because it is extremely difficult to achieve uncertainties of $0.01 \%$ by determining the mass fraction of the main com- ponent directly by a primary method, we generally applied the indirect "difference method". Therefore it was necessary to determine the contents of all possible impurities (including gases and nonmetals). The sum of all relevant impurities was subtracted from $100 \%$ to calculate the purity of the main component. An important aspect is: The purer the material, the lower is the uncertainty achieved for the main component when the "difference method" is used.

Another special feature is that only direct analytical methods, without matrix separation or trace enrichment, can be usefully considered because of the great variety of pure substances and analytes (almost the entire periodic table).

\section{Experimental Details}

\subsection{Reagents and sample preparation}

Samples were analyzed after surface cleaning and -in the case of determination of metallic analytes- by a dissolution with diluted highly purified acids under clean atmosphere conditions using different analytical methods. Reagents, sample pretreatment and dissolution using liquid sample methods (mainly ICP mass spectrometry (ICP MS) and atomic absorption spectrometry (AAS)) are described elsewhere. ${ }^{1)}$

ICP mass spectrometry with laser ablation (LA ICP MS) was calibrated in case of copper analysis with pressed pellets of Cu-powder (m5N; alfa Johnson-Matthey). The pellets had been made from copper powder doped with multi-element standard solutions. The mass fractions of the 40 analytes in the pellets were $0,0.01$ (0.001 for rare earth elements, "RE"), ... up to 100 (10 for RE) $\mathrm{mg} / \mathrm{kg}$. The doping of the powder was carried out without any contact between the doping solutions and the walls of the vessels to exclude losses of analytes. The doped powder was dried by IR-radiation in a laminar flow bench and was homogenized in plastic vessels (with plastic balls) in a mixing apparatus Mixer/Mill (Spex Industr.). The mixture was pressed into steel rings with a $10 \mathrm{~mm}$ inner diameter under a pressure of $100000 \mathrm{MPa} / \mathrm{m}^{2}$ for $10 \mathrm{~min}$.

\subsection{Analytical methods}

The methods used including problems of selecting the appropriate matrix concentration is reported in detail elsewhere. $^{1-5)}$ Therefore only a short information summary is given in this article.

A scheme of the methods used for certification of Type A materials is shown in Fig. 3. Low mass fractions of "metallic" impurities were preferably determined by ICP mass spectrometry using a high resolution sector field mass spectrometer (HR ICP MS). The advantages of this method for the described task are: its broad element coverage with fast multi-element capability, its high selectivity in high resolution mode ${ }^{6-8)}$ and its high sensitivity when low resolution mode was used. ${ }^{3)}$ The spectrometer used was an "Element" (Finnigan MAT) with nickel inlet cones. The nebulizer gas flow rate was optimized for each procedure. In most cases analyses were carried out in low resolution mode $(R=300)$. To overcome or to clear up spectral interferences, higher resolution modes $(R=3000$ or 8000$)$ were employed. The matrix concentration was typical $0.001 \mathrm{~kg} / \mathrm{L}$. For further experimental details see. ${ }^{1)}$

In specific cases two special sample introduction tech- 


\section{Scheme of certification of ultrapure metals as primary calibration substances (Type A)}

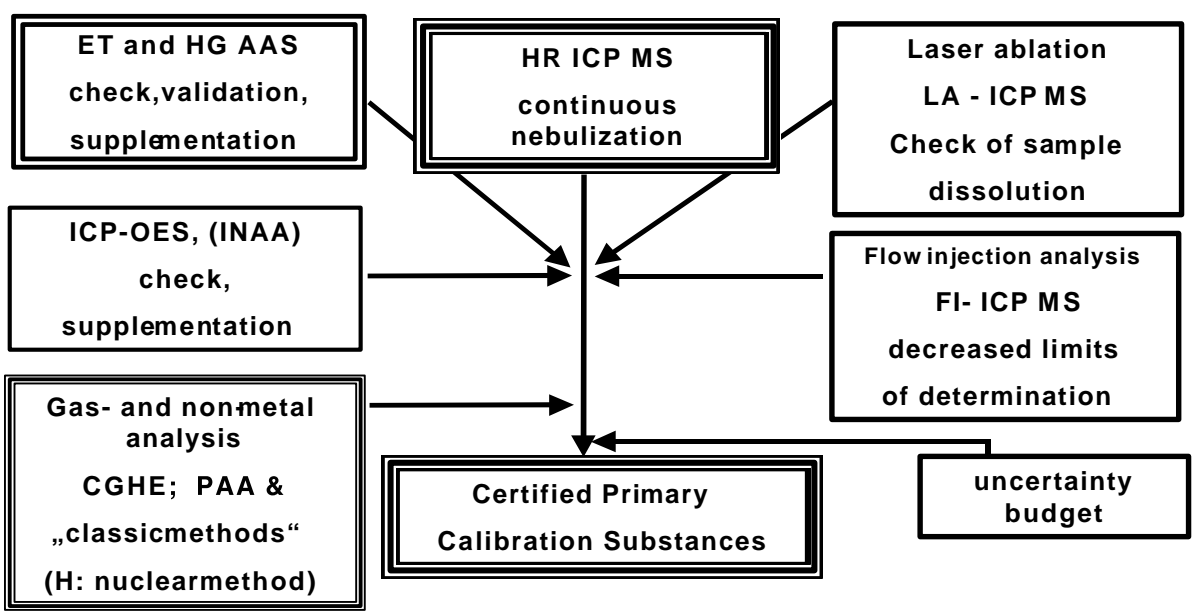

Fig. 3 Scheme of certification of primary calibration substances of Type A.

niques for ICP MS were applied (Fig. 3, right) additionally to the main technique of continuous nebulization. Flow injection analysis (FIA) was used for higher matrix concentrations (up to $0.005 \mathrm{~kg} / \mathrm{L}$ ). The spectrometer was connected with a Perkin-Elmer 400 FIA-system using a $200 \mu \mathrm{L}$ sampling loop. Using FIA lower detection limits in the solid sample could be attained.

A Finnigan MAT laser probe with a wavelength of $266 \mathrm{~nm}$ was used for clearing up potential losses or contamination during sample preparation by direct solid sample analysis. The analytical parameters were: $25 \mathrm{~mJ}$ laser energy, a shot frequency of $5 \mathrm{~Hz}, 15$ shots at each spot positioned in a linear trace. The nominal spot diameter was $200 \mu \mathrm{m}$ and the distances of spot centers in the trace were $30 \mu \mathrm{m}$.

Other methods than ICP MS are shown in Fig. 3, left side. The complemental basic method used for the determination of a selected number of metallic trace elements was atomic absorption spectrometry (AAS). Although it does not have an extensive simultaneous multi-element capability it is useful because of its high detection power and excellent selectivity. Electrothermal atomization AAS (ET AAS) (spectrometers: Perkin-Elmer Zeeman 5100 PC and Perkin-Elmer SIMAA6000) was used to confirm many results of HR ICP MS. The additional information was especially important in case of interferences in ICP MS. Also for light elements lower limits of determination compared to HR ICP MS were obtained. The used matrix concentration (often between $0.005-0.01 \mathrm{~kg} / \mathrm{L}$ ) was higher than used by HR ICP MS. Most of the analytes were atomized from a glassy carbon platform positioned in a pyrolytically coated graphite tube. A Perkin-Elmer SIMAA6000/AS-72 was used in combination with a standard THGA graphite tube with an integrated pyrolytically coated platform. AAS with hydride generation (HG AAS) was applied to achieve low limits of determination for hydride forming analytes. In this case the ET AAS was combined with the Perkin-Elmer FIAS-400/AS-90.
When higher levels of analyte mass fractions in Type A materials were observed, confirmation by more than two methods was necessary. In these cases inductively coupled plasma optical emission spectrometry (ICP-OES) (spectrometer: JY-70Plus, Jobin Yvon) or instrumental neutron activation method (INAA) (carried out by BAM specialists using the nuclear research reactor of Hahn-Meitner-Institute, Berlin) were used.

Carrier gas hot extraction method and combustion method combined with different detection methods were used for the determination of $\mathrm{O}$ and $\mathrm{N}$ and of other non-metals $(\mathrm{C}, \mathrm{S}){ }^{5}$ ) The results are of high relevance for certifying primary substances of Type A materials. For detection, the "classic" method (instruments by Leco) was used as well as the photon activation analysis (PAA) (instrument: BAM linear accelerator). Using the "classic" method, the contents found are the sum of bulk gas contents and surface contents after cleaning. The bulk contents alone are determined after a cleaning procedure by PAA. The latter method therefore gives a lower limit for the result of the "classic" method. The calibration of both methods was carried out by using pure compounds like oxides or nitrates (partly as dried solutions) to achieve a metrological traceability. For the determination of hydrogen a nuclear reaction method was developed and employed at BAM.

\subsection{Special techniques}

The possibility to check results for potential losses and contamination during sample preparation is given by HR ICP MS in combination with laser ablation. As for all direct solid sample techniques the calibration of this analytical method is complicated. Therefore the obtained results often bear semiquantitative character. On the other hand a very good possibility is given to check for elements which could be lost by adsorption or by evaporation and for contamination caused by wet chemical sample handling and sample preparation. ${ }^{9)}$ 
Some authors have applied the method by using pressed pellets as samples for the analysis of geological ${ }^{10,11)}$ or organic material. ${ }^{12)}$ Regarding ultra pure metals the method has been applied successfully in case of ultra pure copper ${ }^{4)}$ and it will be tested and applied for other materials too. In the following section some experimental details are presented concerning the calibration of the analytical procedure (LA ICP MS) applied to the analysis of a high-purity copper material.

Pressed pellets from copper powder ( $55 \mathrm{~N}$, grain size about $5-10 \mu \mathrm{m}$ ) had to be prepared, because calibration sample sets of pure copper, containing the relevant large number of analytes and graduated to such low levels $(0.001 \ldots 100 \mathrm{mg} / \mathrm{kg})$ of analyte mass fractions are not available. To achieve an appropriate micro homogeneity of the analytes in the pellets doping with liquids was preferred to doping with powders containing compounds of analytes. The copper powder used as a basic material for preparation of pellets was ideal for this purpose, because of its porous surface allowing the salt residues to have a good adhesion.

In process of doping the problem was not to destroy the porous grain surface by the acids contained in the solutions for stabilizing the analyte concentrations. Therefore acid concentrations were adjusted as low as possible and the time for drying was kept low by IR heating the sample. With the short drying time the formation of singular crystals not being in adhesion with grains of the copper powder was avoided. This was confirmed by investigations with secondary ion mass spectrometry (SIMS). ${ }^{1,9)}$ In Fig. 4 the original and the doped copper grains are mapped in different magnifications using SEM. It is obvious that neither additional grain clusters had formed nor damages of the pore structure or grain surface are

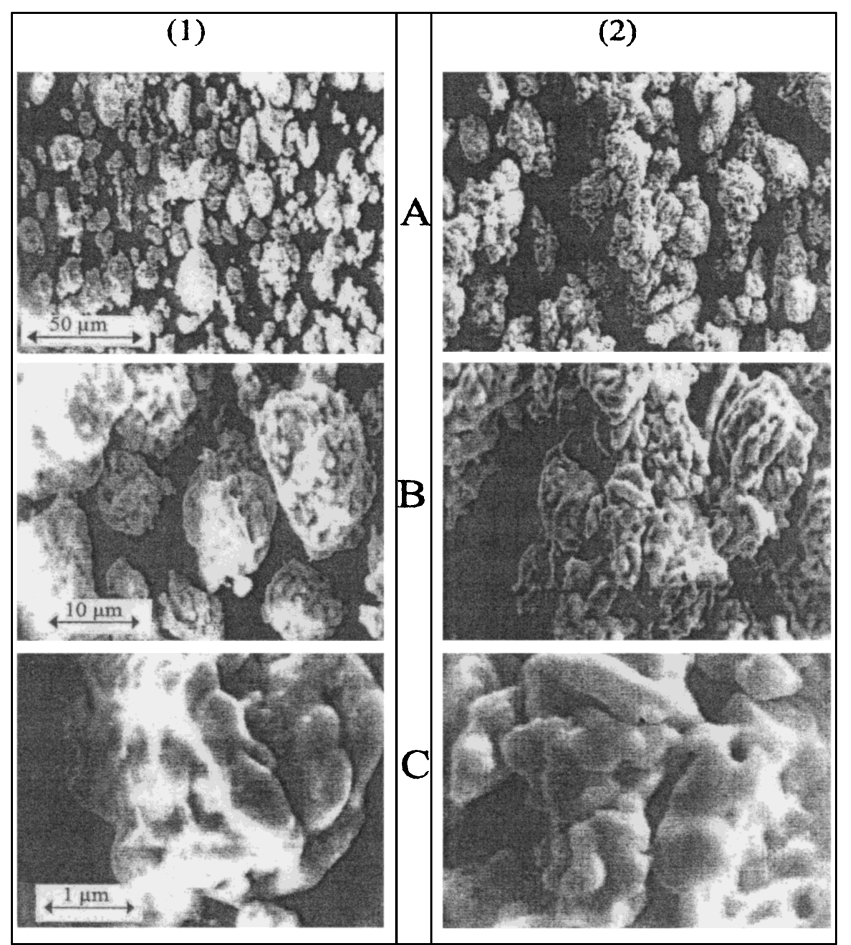

Fig. 4 SEM micrographs of the copper powder used for preparing solid calibration samples for laser ablation ICP MS before (2) and after (1) doping with multielement solutions followed by drying and homogeneizing the sample. Doped analyte contents were $100 \mathrm{mg} / \mathrm{kg}$. Different magnifications (A-C) were applied. detectable as a result of sample doping. This is a good basis to prepare pressed copper powder samples for LA ICP MS.

As explained above the diameter of laser shots in a trace of shots was $200 \mu \mathrm{m}$. Comparing with Fig. 5B one can conclude that much more than one cluster of pressed grains were covered by the dimension of one shot.

Another important question was the similar ablation behaviour of real solid samples and pressed samples used for calibration. A stronger ablation from pressed pellets than from solid samples was found by SEM micrographs. ${ }^{1,9)}$ Additionally a selective evaporating of analytes from the pellets was observed by investigation of analytical signals and by SIMS micrographs of ablated sample areas. That is why analytical comparability of calibration pellets and compact pure copper material is difficult. However, by using internal standardization $\left(\mathrm{CuAr}^{+}\right.$-ion intensity) and correction factors calculated from analytical results achieved with CRMs of known content, it was possible to achieve a rather good semi-quantitative agreement of the results by LA ICP MS and obtained by conventional ICP MS. The results differed between 5 and $15 \%$. The limits of determination (LODs) were in the range of $0.009 \mathrm{mg} / \mathrm{kg}$ (Yb174) to $2.3 \mathrm{mg} / \mathrm{kg}$ (Na23). The LODs were $<0.04 \mathrm{mg} / \mathrm{kg}$ for most analytes with atomic masses $>138$ and they were $<0.5$ for most analytes with atomic masses $>51$. The linearity of the calibration curves was very good in the range of analyte contents from $1 \mathrm{mg} / \mathrm{kg}$ (and higher) down to $0.001 \mathrm{mg} / \mathrm{kg}$ in the pressed pellets. The coefficients of correlation of the linear calibration functions were in most cases $>0.995$. For analytes with atomic masses $>138$ most coefficients of correlation were $>0.998$. In summary LA ICP MS could be used for checking losses and contamination occurring during sample pre-treatment of the liq-
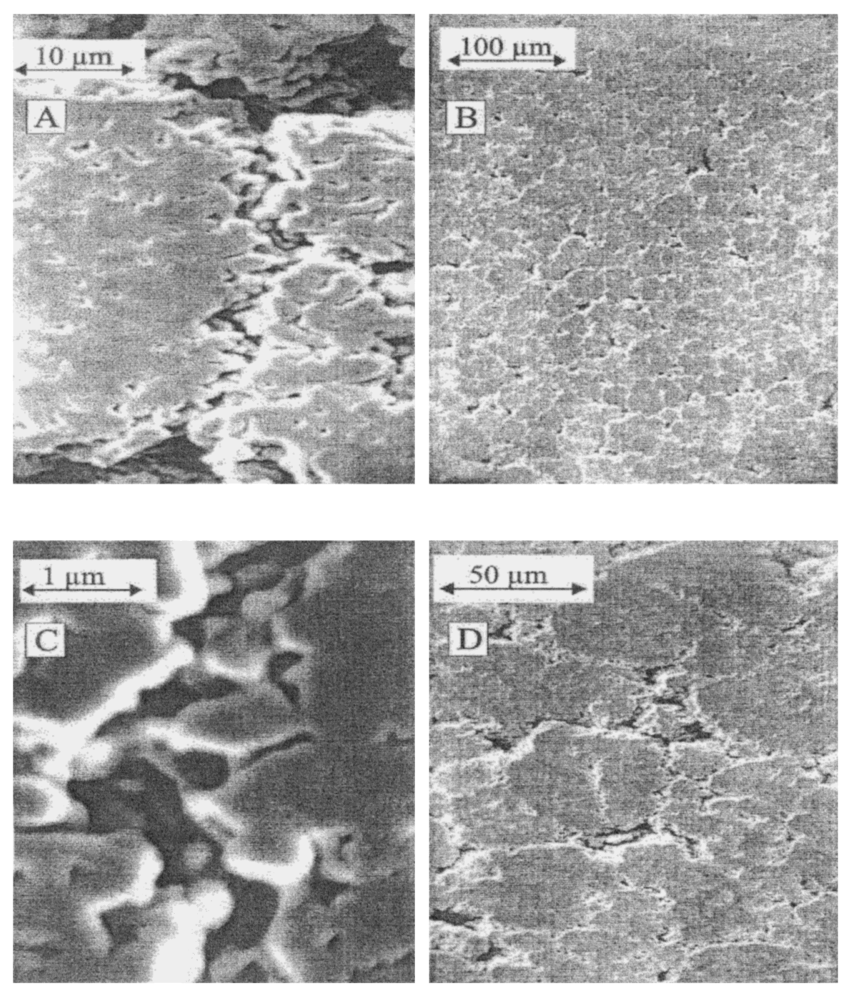

Fig. 5 SEM micrographs of pressed copper powder sample in different magnifications A-D; doped analyte contents $100 \mathrm{mg} / \mathrm{kg}$. 
uid samples used for the ICP MS in conventional sampling mode.

The problem of contamination in the sample pre-treatment procedures for laser ablation itself had been studied before by preparing series of pressed pellets: one from pure copper powder (for checking contamination from mechanical pretreatment) and one from copper powder, doped with the diluted acids not containing doping analytes (for checking additional contamination from doping process). The observed contamination was minimized by optimizing preparation conditions and media. The remaining analytical ICP MS counting rates which were a result of contributions from contamination and from non-characteristic spectral background were used for correcting the measured signals of the samples.

Another special technique used in combination with ICP MS was the flow injection analysis (FIA). Using copper and gallium as examples it had been shown that higher matrix concentrations were corresponding with lower LODs in the solid samples, compared with LODs obtained with lower matrix concentrations in solution. ${ }^{2)}$ Generally, the LODs (related to solid samples) decreased with increasing matrix concentrations $(0.0001 \mathrm{~kg} / \mathrm{L}, 0.001 \mathrm{~kg} / \mathrm{L}, 0.002 \mathrm{~kg} / \mathrm{L}, 0.005 \mathrm{~kg} / \mathrm{L})$. This correlation was observed in spite of the occurring matrix depressions, which were overcompensated by the higher conversion factor from liquid to solid samples for higher matrix concentrations. However, this correlation cannot be used as an advantage in the normal ICP MS mode with continuous nebulization because of strong drifts of signals occurring mainly as a consequence of deposition of matrix material on the cones. ${ }^{2,3)}$ When using flow injection analysis (FIA) the loading time of the cones of the spectrometer inlet system can be shortened because of the transient signals obtained by this technique. Therefore the matrix induced drift of signals measured over some analytical cycles was lower using FIA technique. As a consequence, higher matrix concentrations could be used leading to lower limits of determination relative to the solid sample even with a resulting greater noise of the signal. Low limits of determination were observed with higher matrix concentration of the matrix copper (up to $0.004 \mathrm{~kg} / \mathrm{L}$ ) as well as a good agreement between contents determined by FIA ICP MS with those of continuous nebulization. Therefore it is of advantage to use the special technique, when lower LODs than those achieved with continuous nebulization and normally used matrix concentrations (up to $0.001 \mathrm{~kg} / \mathrm{L}$ ) are necessary to attain.

\section{Results of Certification}

\subsection{Primary copper materials}

The certification of some Type A materials was carried out using the different analytical methods and special techniques as mentioned above. For copper the starting material was a nominal $\mathrm{m} 4 \mathrm{~N}$ high purity copper material (alfa Johnson Matthey) as spheres (diameters $\sim 1 \mathrm{~cm}$ ). All the relevant metallic trace concentrations were determined using diluted aliquots of solutions prepared from different copper spheres. The results were compiled and evaluated. The values of mass fractions of 73 trace elements in the copper material were determined to be either above the limits of determination (LOD) or below so called "limit-values" (LV), whereas
$\mathrm{LV}>=\mathrm{LOD}$.

There were 16 of the 73 investigated trace elements measured to be above the LODs (Ag, As, Bi, C, Ca, Cr, Fe, N, Ni, $\mathrm{O}, \mathrm{Pb}, \mathrm{S}, \mathrm{Sb}, \mathrm{Se}, \mathrm{Si}, \mathrm{Sn}$ ) and the rest was found to have mass fractions below LVs. The highest mass fraction was determined for silver with $11.2 \mathrm{mg} / \mathrm{kg}$. This value was determined from results of HR ICP MS $(11.4 \mathrm{mg} / \mathrm{kg})$, ET AAS $(11.6 \mathrm{mg} / \mathrm{kg})$, ICP-OES $(11.3 \mathrm{mg} / \mathrm{kg})$ and INAA $(10.5 \mathrm{mg} / \mathrm{kg})$.

The mass fractions of the halogens, the noble gases and most of the radioactive elements were not determined. These trace elements were assumed to be "not relevant" to the final value of the mass fraction of copper and its calculated uncertainty. The calculation of the mass fraction of copper in this material and its uncertainty was carried out as follows. For the mass fraction of copper itself the mass fractions of all traces determined above LODs were added up $(=24.79 \mathrm{mg} / \mathrm{kg})$ as well as the half values of LVs for the traces determined below LVs $(=7.54 \mathrm{mg} / \mathrm{kg})$. Half values of LVs were used because these are the most probable values for the mass fractions which had been determined below LV. Both contributions were added $(=32.33 \mathrm{mg} / \mathrm{kg})$ and then subtracted from $100 \%$. Thus a value of $99.9968 \%$ for the mass fraction of copper was calculated.

For calculating the uncertainty of this result the following assumptions were made. The single values determined above LODs have relative uncertainties of $30 \%$ and the single values below "LVs" have relative uncertainties of $100 \%$. From that, it follows that all values determined below LVs were assumed to have mass fractions of (LV/2 $\pm \mathrm{LV} / 2)$, thus the entire interval $(0 \ldots L V)$ is covered by the uncertainty interval. By experience the real values for the uncertainties of elements found above LODs are mostly lower than the assumed $30 \%$. Thus an upper limit for the entire uncertainty should be estimated. By quadratic addition of single values the contribution of the "above LOD values" to the final uncertainty was calculated to be $3.9 \mathrm{mg} / \mathrm{kg}$ - and the contribution of the "below LV values" was calculated to be $2.6 \mathrm{mg} / \mathrm{kg}$. By quadratic addition of both contributions a total value of $4.7 \mathrm{mg} / \mathrm{kg}$ (or of about $0.0005 \%$ ) was calculated as the uncertainty for the copper mass fraction. The certified value of the mass fraction in the BAM A-primary-Cu 1 material is therefore $(99.9968 \%+-0.0005 \%)$. In this way an extremely low uncertainty was achieved, the value of which is more than one order of magnitude lower than the upper limit of the target uncertainty $(0.01 \%$, see above), demonstrating the great capabilities of the "difference method" used. This was possible because of the high purity of the material resulting in small absolute single uncertainties and, therefore, a small relative uncertainty of the main component. By additional calculations it could be demonstrated that even larger uncertainties stated for single trace determinations had only a marginal enlarging influence on the final total uncertainty.

In Fig. 6 a comparison was made between the permitted uncertainty of main component $(0.01 \%)$ and four calculated results for the uncertainty of the copper content of primary copper A-material, based on different assumptions for their calculation. Independent from the assumed extent of the uncertainties of analyte contents found above LODs (uncertainties $=20 \ldots 50 \%$ of corresponding content) and 


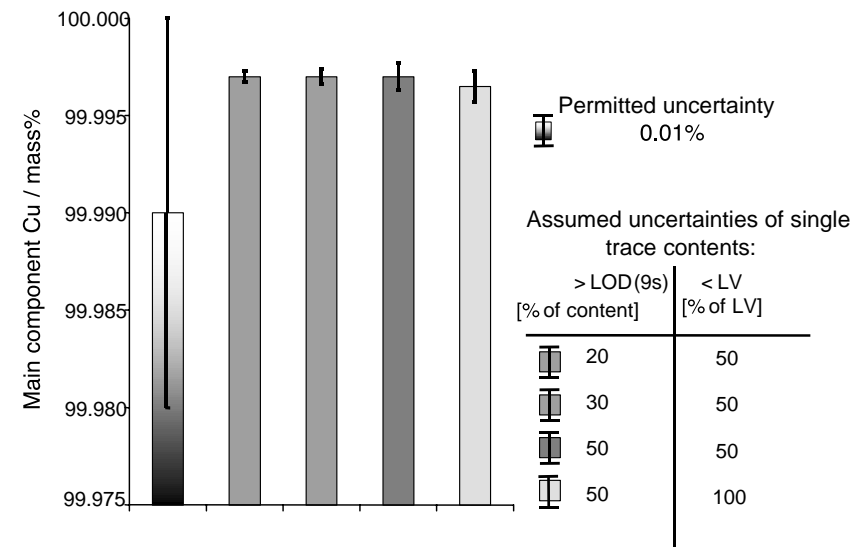

Fig. 6 Comparison of the permitted uncertainty of the final result and calculated uncertainties (on the basis of different assumptions for their calculation; primary copper Type A material).

independent of the assumed uncertainties of analyte contents found below LVs (uncertainties $=50 \ldots 100 \%$ of corresponding LVs) the calculated four final uncertainties of main copper content are similar and much lower than the permitted value of $0.01 \%$.

Additionally to the Type A, a Type B copper material was certified. This material, declared as a m6N material by producer, will be used for metrological problems of matrix matching and is therefore of much higher purity with respect to the metallic analytes. However the opposite is true for the non-metallic analytes $\mathrm{O}$ and $\mathrm{N}$. The mass fraction of oxygen in the Type B material exceeds that of the Type A material by a factor of more than 500. Therefore the oxygen content would give a high contribution to the sum of mass fractions of traces, and to its uncertainty, if this nominal m6N material would be certified as an Type A material: In this case the copper mass fraction would be calculated as $99.944 \%+-0.017 \%$, or about $\mathrm{t} 3 \mathrm{~N} 5$. The uncertainty would exceed the acceptable limit of $0.01 \%$. This is an evidence for the thesis that the nominal (metal based) purity value supplied by the producer doesn't give any information about the real (total) purity of the metal and that it has to be determined by measurement. However, the Type B material is very useful for metrological questions of matrix matching because of its very low metallic impurities. The highest mass fraction is for silver with $0.33 \mathrm{mg} / \mathrm{kg}$.

\subsection{Primary iron material}

Two iron materials of Type A were certified. As a starting material for the certification of the primary calibration substance "BAM A-primary-Fe 1" a pure iron material (alfa Johnson Matthey) was used having a nominal purity of $\mathrm{m} 3 \mathrm{~N}$ and being declared to have low gas content. For this material an iron mass fraction of $99.966 \%+-0.009 \%$ was certified.

This material could be accepted as an Type A material but the uncertainty for the main component, which is mainly due to a rather high level of oxygen $(288 \mathrm{mg} / \mathrm{kg})$, is not much below the aimed upper level of uncertainty of $0.01 \%$.

To demonstrate and to test another way of certifying a Type A material a certified reference material $(\mathrm{CRM})^{13)}$ was used. This one was a pure iron CRM of the EURONORM group (EURONORM CRM 098-1) certified for six analytes and with an indicated value for one analyte. The certification as an primary calibration material of Type A was carried out by determining the other relevant analytes $(>60)$ of the periodic table. For this "BAM A-primary-Fe 2" material an iron mass fraction of $99.987 \%+-0.001 \%$ was certified. One can conclude that by this way of using a CRM as a starting material an uncertainty for the iron mass fraction of about one order of magnitude lower than the upper limit could be achieved.

To validate the results of the main methods (HR ICP MS and ET AAS) used for certification of metallic analytes in pure primary iron materials of Type A, a comparison of results of the methods was made for analytes found above LODs (Al, Ca, Co, Cr, Cu, Ga, Ge, Mn, Mo, Ni, Pb, Sn, Zn). A very good agreement between results of both methods could be stated in most cases.

\subsection{Primary lead material}

The starting material for the Type A lead material had a very high "metal" purity of m5N5. Very low metallic trace contents and, additionally low contents of non-metallic analytes were determined. As for the copper Type A material this was a basis very favourable for the certification of lead Type A material. In Fig. 7 the results are shown in form of a periodic table.

Only mass fractions of 6 elements were found above LODs. The certification was carried out as described for copper. The certified lead mass fraction was $(99.9982+-0.0006) \%$. The uncertainty for the main component is much lower than the permitted uncertainty of $0.01 \%$. In Fig. 8 an example is represented, demonstrating that in special cases only the measurement in higher resolutions allowed to determine some elements without interferences.

\subsection{Other materials, outlook}

Two gallium materials, one of Type A and one of Type B, are under development. Both materials are of high purity. For the Type A material a low uncertainty of the main component is expected. The same is expected for a pure tin material of Type A which is under investigation. In the gallium material of Type A highest contents of non-metallic traces are in the region of some $\mathrm{mg} / \mathrm{kg}$. Mass fractions of most metallic traces are at sub $\mathrm{mg} / \mathrm{kg}$ level. Further primary calibration materials will be certified in future.

\section{Conclusion}

A system of primary reference materials is under development at BAM. This system represents national standards for elemental analysis underlined by a contract with the German sister institute PTB. A "difference method" was found to give excellent results for certifying mass fractions of the main contents in pure primary materials of Type A (used for analyte calibration). The laboratory is accredited for the trace analytical methods used by DAP Deutsches Akkreditierungssystem Prüfwesen $\mathrm{GmbH}$ according to the European Standard EN 45001 (in future ISO/IEC 17025). ${ }^{14)}$ A short documentation of the entire difference method is given in the catalogue of reference procedures provided by BAM. ${ }^{15)}$ An uncertainty of the mass fraction of the main component much lower than $0.01 \%$ can be attained by applying the difference method. For this 


\begin{tabular}{|c|c|c|c|c|c|c|c|c|c|c|c|c|c|c|c|c|c|}
\hline & & & & & & & & & & & & & & & & & \\
\hline & & & & & & & & & & & 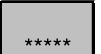 & not y & et dete & ermine & & & \\
\hline & $a b c$ & ove lim & nit of $d e$ & etermin & Iation L & -OD & & & & & & deter & minatic & ion not & relevar & & \\
\hline & belc & ow limit & it value & LV & & & & & & & $?$ & deter & minatic & on not & relevar & nt? & \\
\hline$\underset{* \star * \star * \star}{\mathbf{H}}$ & & & & $\begin{array}{l}\text { BAM } \\
\text { candida }\end{array}$ & ate mater & $\begin{array}{l}\text { - Prin } \\
\text { rial: Alfa }\end{array}$ & $\begin{array}{l}\text { nary- } \\
\text { Johnson }\end{array}$ & $\begin{array}{l}\text { Pb 1" } \\
\text { Matthey }\end{array}$ & m5N5 & & & & & & & & $\mathrm{He}$ \\
\hline $\begin{array}{c}\mathrm{Li} \\
<0.1\end{array}$ & $\begin{array}{c}\mathrm{Be} \\
<0.1\end{array}$ & & 11 mass & fractio & ns in $r$ & $\mathrm{ng} / \mathrm{kg}$ & ( & & & & & $\begin{array}{c}\text { B } \\
<1.0\end{array}$ & $\begin{array}{c}C \\
5.2\end{array}$ & $\begin{array}{c}\mathrm{N} \\
0.4\end{array}$ & $\begin{array}{c}0 \\
3.8\end{array}$ & $F$ & $\mathrm{Ne}$ \\
\hline $\begin{array}{l}\mathrm{Na} \\
<0.7\end{array}$ & $\begin{array}{c}\mathrm{Mg} \\
<0.05\end{array}$ & & Sum & $<L V$ & $\begin{array}{l}=10 . \\
=6 .\end{array}$ & $85+-2$ & $2.9 \mathrm{mg}$ & & & & & $\begin{array}{c}\text { Al } \\
<0.5\end{array}$ & $\begin{array}{c}\mathrm{Si} \\
<5.0\end{array}$ & $\begin{array}{c}\mathbf{P} \\
\star \star \star \star \star \star\end{array}$ & $\begin{array}{c}S \\
0.0\end{array}$ & $\begin{array}{l}\mathrm{Cl} \\
?\end{array}$ & $\begin{array}{c}\mathrm{Ar} \\
?\end{array}$ \\
\hline $\begin{array}{c}\mathrm{K} \\
<0.5\end{array}$ & $\begin{array}{c}\mathrm{Ca} \\
<0.5\end{array}$ & $\begin{array}{c}\text { Sc } \\
<0.3\end{array}$ & $\begin{array}{c}\mathrm{Ti} \\
<0.05\end{array}$ & $\begin{array}{c}\mathrm{V} \\
<0.05\end{array}$ & $\begin{array}{c}\mathrm{Cr} \\
<0.05\end{array}$ & $\begin{array}{c}\mathrm{Mn} \\
<0.05\end{array}$ & $\begin{array}{l}\mathrm{Fe} \\
<0,2\end{array}$ & $\begin{array}{c}\text { Co } \\
<0.02\end{array}$ & $\begin{array}{c}\mathrm{Ni} \\
0.74\end{array}$ & $\begin{array}{c}\mathrm{Cu} \\
0.27\end{array}$ & $\begin{array}{c}\mathrm{Zn} \\
<0.2\end{array}$ & $\begin{array}{c}\mathrm{Ga} \\
<0.3\end{array}$ & $\begin{array}{c}\mathrm{Ge} \\
<0.05\end{array}$ & $\begin{array}{c}\text { As } \\
<0.05\end{array}$ & $\begin{array}{c}\mathrm{Se} \\
<1.0\end{array}$ & $\mathrm{Br}$ & $\mathbf{K r}$ \\
\hline \begin{tabular}{|c|}
$\mathbf{R b}$ \\
$<0.01$ \\
\end{tabular} & $\begin{array}{c}\mathrm{Sr} \\
<0.001 \\
\end{array}$ & $\begin{array}{c}Y \\
<0.005 \\
\end{array}$ & $\begin{array}{c}\mathrm{Zr} \\
<0.001 \\
\end{array}$ & $\begin{array}{c}\mathrm{Nb} \\
<0.001 \\
\end{array}$ & $\begin{array}{c}\text { Mo } \\
<0.01 \\
\end{array}$ & Tc & $\begin{array}{c}\mathrm{Ru} \\
<0.01 \\
\end{array}$ & $\begin{array}{c}\mathbf{R h} \\
<0.01 \\
\end{array}$ & $\begin{array}{c}\mathrm{Pd} \\
<0.01 \\
\end{array}$ & $\begin{array}{c}\mathrm{Ag} \\
0.34 \\
\end{array}$ & $\begin{array}{c}\mathrm{Cd} \\
<0.01 \\
\end{array}$ & $\begin{array}{c}\text { In } \\
<0.005 \\
\end{array}$ & $\begin{array}{c}\text { Sn } \\
<0.1\end{array}$ & \begin{tabular}{|c|}
$\mathrm{Sb}$ \\
$<0.005$ \\
\end{tabular} & \begin{tabular}{|c|}
$\mathrm{Te}$ \\
$<0.02$ \\
\end{tabular} & I & $\mathrm{Xe}$ \\
\hline \begin{tabular}{|c|} 
Cs \\
$<0.001$
\end{tabular} & $\begin{array}{c}\mathrm{Ba} \\
<0.003 \\
\end{array}$ & La-Lu & \begin{tabular}{|c|}
$\mathrm{Hf}$ \\
$<0.001$ \\
\end{tabular} & \begin{tabular}{|c|}
$\mathrm{Ta}$ \\
$<0.001$ \\
\end{tabular} & $\begin{array}{c}\mathrm{W} \\
<0.05 \\
\end{array}$ & $\begin{array}{c}\mathbf{R e} \\
<0.001 \\
\end{array}$ & $\begin{array}{c}\text { Os } \\
<0.001 \\
\end{array}$ & \begin{tabular}{|c|} 
Ir \\
$<0.005$ \\
\end{tabular} & $\begin{array}{c}\mathrm{Pt} \\
<0.05 \\
\end{array}$ & $\begin{array}{c}\mathrm{Au} \\
<0.2 \\
\end{array}$ & $\begin{array}{l}\mathrm{Hg} \\
<0.2 \\
\end{array}$ & $\begin{array}{c}\mathrm{TI} \\
<0.2 \\
\end{array}$ & $\mathbf{P b}$ & $\begin{array}{c}\mathrm{Bi} \\
<2.0 \\
\end{array}$ & Po & At & $\mathbf{R n}$ \\
\hline $\mathrm{Fr}$ & $\mathbf{R a}$ & $A c-L r$ & & Total & trace & 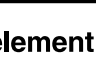 & conter & nt: $(0.00$ & $01075+$ & 0.0006 & $685) \%=$ & $=0.0017$ & $6 \%$; un & ncertain & nty: 0,00 & $006 \%$ & \\
\hline & $\frac{\text { certifie }}{\mathrm{Pb}-\text { con }}$ & tent: & $\begin{array}{c}\text { La } \\
<0.001 \\
\end{array}$ & $\begin{array}{c}\mathrm{Ce} \\
<0.001\end{array}$ & $\begin{array}{c}\mathrm{Pr} \\
<0.001 \\
\end{array}$ & $\begin{array}{c}\mathbf{N d} \\
<0.005 \\
\end{array}$ & Pm & $\begin{array}{c}\mathrm{Sm} \\
<0.005\end{array}$ & \begin{tabular}{|c|}
$\mathrm{Eu}$ \\
$<0.001$ \\
\end{tabular} & $\begin{array}{c}\text { Gd } \\
<0.001 \\
\end{array}$ & \begin{tabular}{|c|} 
Tb \\
$<0.001$ \\
\end{tabular} & \begin{tabular}{|c|} 
Dy \\
$<0.001$ \\
\end{tabular} & $\begin{array}{c}\text { Ho } \\
<0.001\end{array}$ & $\begin{array}{c}\text { Er } \\
<0.001 \\
\end{array}$ & \begin{tabular}{|c|}
$\mathrm{Tm}$ \\
$<0.001$ \\
\end{tabular} & \begin{tabular}{|c|}
$\mathrm{Yb}$ \\
$<0.001$ \\
\end{tabular} & $\begin{array}{c}\mathrm{Lu} \\
<0.001 \\
\end{array}$ \\
\hline & $\begin{array}{r}99.998 \\
+-0.00 \\
\end{array}$ & $\frac{32 \%}{06 \%}$ & $A C$ & $\begin{array}{c}\text { Th } \\
<0.001\end{array}$ & $\begin{array}{c}\mathrm{Pa} \\
<0.001\end{array}$ & $\begin{array}{c}U \\
<0.001\end{array}$ & $N p$ & $\mathrm{Pu}$ & $\mathrm{Am}$ & $\mathrm{Cm}$ & \begin{tabular}{|l|} 
Bk \\
\end{tabular} & \begin{tabular}{l|}
$\mathbf{C f}$ \\
\end{tabular} & Es & $\mathrm{Fm}$ & Md & \begin{tabular}{|l|} 
No \\
\end{tabular} & $\mathrm{Lr}$ \\
\hline
\end{tabular}

Fig. 7 Certification results for the primary lead calibration material of Type A "BAM A-Primary-Pb 1".

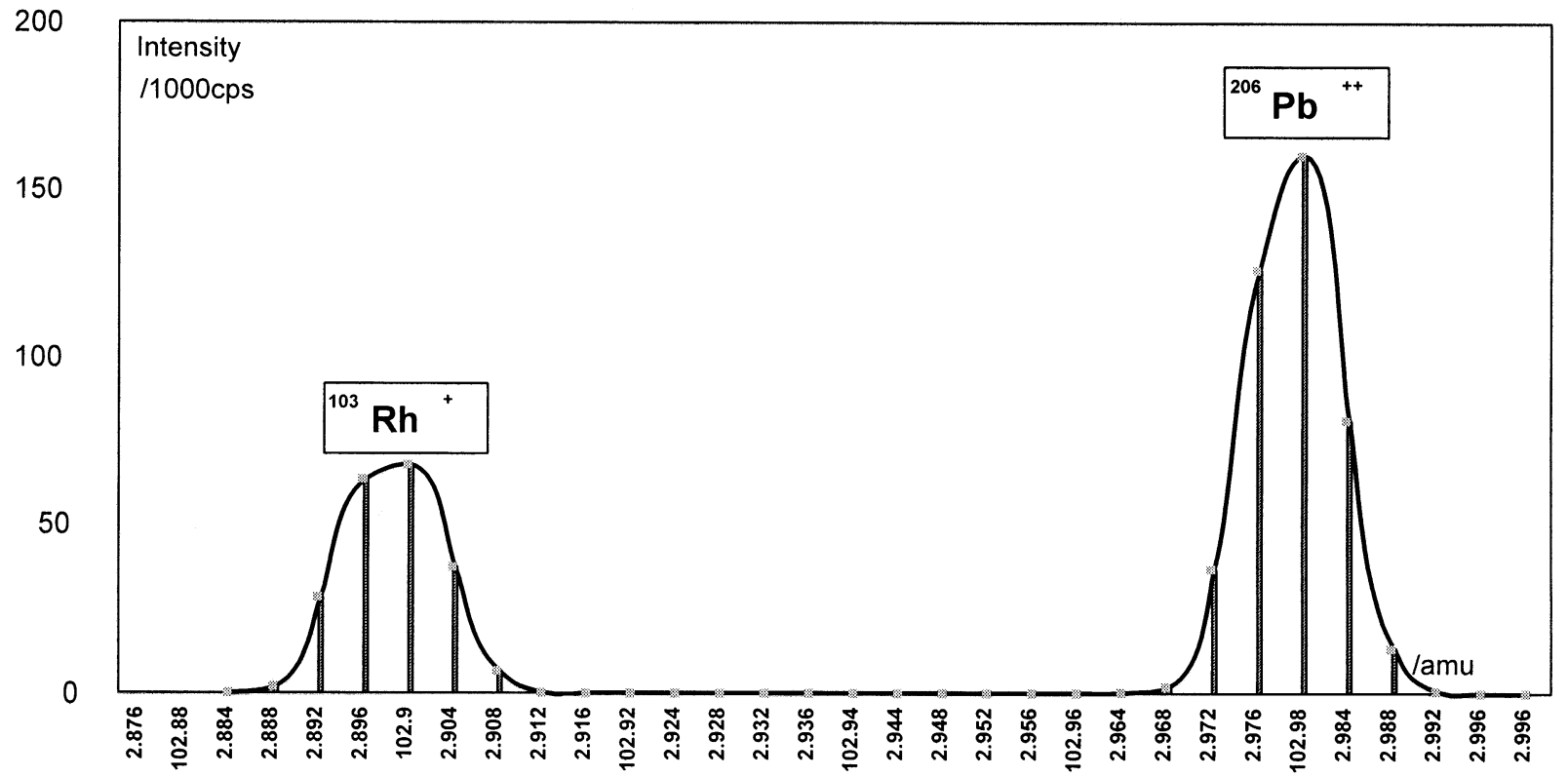

Fig. 8 Certification of "BAM A-Primary-Pb 1". Example for a resolved matrix interference by higher mass resolution $(r=3000)$ : Determination of $\mathrm{Rh}(10 \mathrm{ng} / \mathrm{mL})$ in lead solution $(1000 \mathrm{mg} / \mathrm{L})$. The interference on the mass of the monoisotopic analyte by double charged matrix ions is resolved.

it is necessary to use a starting material which has low contents of metallic and non-metallic impurities. A distinction between Type A material and Type B (used for problems of matrix matching) is useful and related to their application. In some cases it is possible not to start from commercially available high purity materials but from pure CRMs for certifying Type A materials. High oxygen contents are sometimes problematic and should therefore be checked in the beginning of the certification procedure. The combination of different ap- propriate trace and ultra trace analytical methods is necessary for a successful certification procedure at high metrological level.

\section{Acknowledgements}

For their contributions to gas and nonmetal analysis the authors thank Dr. H. Kipphardt, Dr. S. Recknagel, Dr. K. A. Meier and co-workers as well as Dr. M. Hedrich, Th. Dudzus 
and co-workers and Dr. H.-P. Weise and co-workers, and Dr. A. Berger for carrying out determinations by INAA. For contributions to other determinations and to sample preparation we would like to thank A. Dette, N. Langhammer and S. Zimmer- and Dr. E. Schierhorn for carrying out investigations by scanning electron microscopy.

\section{REFERENCES}

1) R. Matschat, M. Czerwensky, S. Pattberg and H.-J. Heinrich: Phys. Status Solidi (2001) in print.

2) R. Matschat, M. Czerwensky, M. Hamester and S. Pattberg: Fresenius J. Anal. Chem. 359 (1997) 418-423.

3) R. Matschat and M. Czerwensky: Phys. Status Solidi 160 (1997) 567574.

4) S. Pattberg and R. Matschat: Fresenius J. Anal. Chem. 364 (1999) 410416.

5) H. Kipphardt, Th. Dudzus, K. A. Meier, S. Recknagel, M. Hedrich and R. Matschat: Mater. Trans., 43 (2002) 98-100.

6) U. Giessmann and U. Greb: Fresenius J. Anal. Chem. 350 (1994) 186-
193.

7) I. Feldmann, W. Tittes, N. Jakubowski, D. Stuewer and U. Giessmann: J. Anal. At. Spectrom. 9 (1994) 1007-1014.

8) L. Moens, F. Vanhaecke, J. Riondato and R. Dams: J. Anal. At Spectrom. 10 (1995) 569-574.

9) S. Pattberg: "Multielement-Spurenbestimmung in Reinstkupfer mit HR ICP MS”, Ph. D. Thesis, Humboldt-Universität, Berlin (1999) pp. 1141.

10) N. Imai: Analyt. Chim. Acta 235 (1990) 381-391.

11) A. A. Van Heuzen and J. B. W. Morsink: Spectrochim. Acta 46B (1991) 1819-1828.

12) E. Hoffmann, C. Lüdke, H. Scholze and H. Stephanowitz: Fresenius J. Anal. Chem. 350 (1994) 253-259.

13) K. Meyer and R. Matschat: Reference Materials in Materials Testing, in: A. Zschunke (Ed.) Reference Materials in Analytical Chemistry, (Springer-Verlag, Berlin, 2000) pp. 57-165.

14) DAP Deutsches Akkreditierungssystem Prüfwesen GmbH, "Certificate of Accreditation No. DAP-P-02.614-14-97-00, Supplements", Berlin (1997) pp. 13-14.

15) W. Hässelbarth et al.: Testing and chemical analysis, catalogue of reference procedures provided by BAM, (Federal Institute for Materials Research and Testing, Berlin, 2001, ISSN 1617-6634) pp. 12-13. 